\title{
"Decime quien consume a quien" \\ Estudio sobre la creación de disposiciones al consumo de bebidas con alcohol generadas por la publicidad en jóvenes
}

\author{
Christian Dodaro \\ Universidad de Buenos Aires (Argentina)
}

\section{Palabras clave}

Consumo, juventud, publicidad

\begin{abstract}
Resumen
La investigación "El alcohol del encuentro" estudio cuantitativo de bebidas alcohólicas y disposiciones al consumo en jóvenes de 13 a 25 años de la República Argentina durante Mayo -Noviembre de 2014. Beca Carrillo Oñativia" ${ }^{1}$ tuvo como objetivo indagar la influencia de publicidades de bebidas alcohólicas utilizando un método de análisis discursivo en su primera etapa, para la cual se trabajó junto al AFSCA, quien facilitó las pautas publicitarias de mayor repetición en canales de aire. Por otro lado, se realizó en la segunda etapa grupos focales para conocer de qué manera inciden los contenidos resultantes del primer análisis en los jóvenes en las provincias de Tucumán, Córdoba, Chaco, Neuquén, Santa Fe y Mendoza de nuestro país.

Si bien había un recorrido realizado en análisis de diversas publicidades en otras partes del mundo, se desconocía el impacto que tienen las mismas en los jóvenes. La concreción del mismo fue a través de un estudio semiótico en su primera etapa, y grupos focales en la segunda para que pudiéramos entender el modo en que las publicidades construyen disposiciones al consumo en los jóvenes.

Durante la investigación, se intentó cruzar el análisis discursivo de las publicidades de bebidas alcohólicas de mayor difusión con los grupos focales para aproximarnos al modo en el que estos presentan disposiciones al consumo de las bebidas alcohólicas. Presentamos las primeras conclusiones del mismo.
\end{abstract}

Autor para correspondencia: Christian Dodaro. Correo electrónico: dodarix@gmail.com

1 Fueron partícipes de la investigación y redacción del informe Lorena Steimber, Ximena Tobi, Sabrina Castillo, Romina Carsillo, Luis Mota y Valeria Campos 


\section{Introducción}

A nivel mundial, el uso nocivo de alcohol causa el 5.9\% de todas las muertes y el $5.1 \%$ de morbilidad, así como representa un importante factor de riesgo para traumatismos involuntarios y prevalentes en múltiples enfermedades.

La Encuesta de Factores de Riesgo de Enfermedades No Transmisibles del año 2011 advirtió que el consumo regular había aumentado en relación al año 2005 en un $10.7 \%$, y que el grupo etáreo de 18 a 24 años de edad era el segundo grupo de riesgo a nivel nacional. En paralelo, en el año 2011 la Encuesta Nacional sobre Prevalencias de Consumo de Sustancias Psicoactivas indicó que la edad iniciática de ingesta de bebidas con alcohol se situaba en los 13 años, mientras que el Observatorio Argentino de Drogas (SEDRONAR, 2008) señaló que el aumento del consumo de alcohol en menores de 12 a 15 años registraba aumentos significativos, especialmente en los grandes centros urbanos.

Esta investigación se plantea indagar en la vinculación entre las publicidades de bebidas alcohólicas y las disposiciones a su consumo en jóvenes de 13 a 25 años, franja de edad considerada de mayor consumo y en la que se pueden generar estrategias de prevención.

Se utilizaron dos métodos de recolección y análisis de datos. Primero, el análisis discursivo del conjunto de publicidades de las marcas líderes de bebidas alcohólicas de mayor exhibición en los canales de aire de las provincias elegidas. Segundo, a través de grupos focales a nuestra población de 13 a 25 años de las distintas regiones sanitarias del país. La investigación intenta vincular el análisis discursivo de las publicidades de bebidas alcohólicas con los grupos focales con el fin de identificar valoraciones, percepciones y acciones del conjunto de jóvenes.

Tomamos como eje la relación de los y las jóvenes con la cultura y el consumo. En la actualidad encontramos una amplia gama de estudios que abordan con diferentes miradas la relación de los y las jóvenes con el consumo y las prácticas culturales. Algunos toman el consumo de sustancias como parte de esas prácticas culturales. Como una forma en la que los jóvenes hacen lo que pueden con lo que tienen a mano.

En ese mismo período otros estudios comienzan a problematizar la capacidad de agencia de los jóvenes en relación con las formas en las que la publicidad en tanto producto de un sistema basado sobre el capitalismo industrial, genera estrategias de inclusión de las pretendidas prácticas alternativas. ¿Todo es manejable, adquirible? ¿porque todo valor tiene un precio de venta y uno de compra? Como plantea El Indio Solari: ¿"Nike es la cultura"? Es el momento de conversión de la cultura juvenil en artículo de consumo (a principio de los 90). Y desde el consumo y las prácticas sociales relacionadas a formas particulares de reelaborar ese consumo, acompañado de despolitización, diferenciación a través del consumo. Con la inclusión de cool hunters, los cazadores furtivos son siempre cazados por cazadores profesionales. Las marcas nos han marcado dirá Klein (2001).

En ese escenario, este trabajo intenta aportar una continuidad respecto al uso del tiempo libre y los consumos de los jóvenes durante sus salidas nocturnas. El análisis de las publicidades se realiza con el fin de ver cuánto de las propuestas de las mismas es retomado en las prácticas concretas de los jóvenes. A través de ello se intenta continuar las investigaciones respecto a los modos de ser jóvenes que existen actualmente. 
Nuestra pregunta se centra en cómo se relacionan los mensajes publicitarios con el consumo de los jóvenes. Se busca establecer las relaciones significantes entre los discursos publicitarios y las prácticas de consumo de los jóvenes. Ello centrado en cómo problematizar la agencia reflexiva en un espacio regulado por el mercado.

Es por esta razón que analizar las representaciones de los jóvenes, las concepciones que tienen de sus pares, del tiempo libre, del fin de semana, de los valores simbólicos, lugares y situaciones de ingesta de alcohol representados en las publicidades, es fundamental para dar cuenta de dichos procesos que también definen la identidad de la juventud como categoría socio-cultural.

\section{Metodología}

La perspectiva teórica de este trabajo se enmarca dentro de los problemas abordados por el enfoque semiótico y antropológico-cultural. Desde este marco general es posible, además, estudiar el problema de la significación y del sentido como una dimensión central de los fenómenos sociales, respondiendo así al eje de las problemáticas que se plantearon en esta investigación. El abordaje cultural, por otra parte, permite relacionar y articular medios masivos, cultura y sociedad. El interés de la investigación estuvo puesto principalmente en cómo se construye el discurso publicitario para interpelar al destinatario de manera de motivar la conducta de consumo de bebidas alcohólicas, cómo se representa la juventud, entendida como categoría socio-cultural, qué escenarios se presentan, qué situaciones de consumo de dichos productos, qué valores simbólicos se materializan y a través de qué modalidades, etc.

Desde esta perspectiva teórica se pueden comprender los procesos de construcción de sentido. Para ello, se analizaron las propiedades de los discursos publicitarios y los modos de funcionamiento del sentido. Se realizó un análisis relacional: los sistemas de relaciones que todo producto significante mantiene con sus condiciones de producción por una parte, y con sus efectos, por la otra.

En la búsqueda de invariantes en el análisis de las campañas publicitarias, se utilizaron algunas herramientas teóricas que se desarrollarán a continuación:

El estudio de las estrategias discursivas permite definir los contratos de lectura que se establecen en cada campaña. Verón sostiene que la enunciación es el contrato. En consecuencia, el estudio de las modalidades enunciativas, entendido como un nivel de análisis del funcionamiento discursivo, permitió comprender qué es lo distintivo de cada campaña en el medio televisivo de la televisión abierta y de las fanpages. Con el estudio de la enunciación, se pudo describir un nivel de funcionamiento del discurso que permitió conocer cómo se construye la imagen del enunciador, la marca, del destinatario y del nexo entre ambas entidades. El contrato de lectura se define a través del nexo que se establece entre el enunciador y el destinatario a través de distintos elementos: relación texto-imagen, dispositivos de apelación, música, movimientos de cámara, uso de polifonías, tipos de planos, etc. Estos rasgos construyen la relación entre el discurso del soporte y sus destinatarios.

En la instancia del reconocimiento, se efectuaron cuatro grupos focales por provincia, dos representativos de barrios populares y dos de barrios residenciales. Cada uno conformado por alrededor de entre 8 y 10 personas. De esta manera, se articularon ambas instancias para poder responder a los objetivos de la investigación. Para articular ambas instancias: la producción publicitaria sobre el consumo de bebidas alcohólicas generada por las marcas y el discurso de los jóvenes sobre su propia práctica de consumo de alcohol, nos resultó clave el concepto de estilos 
discursivos sociales propuesto por José Luis Fernández, ya que vincula discursos y segmentos sociales, las dos entradas analíticas con que trabajamos. En palabras del autor, el estilo discursivo social se refiere a "la dimensión específicamente textual de la dimensión significante de los fenómenos sociales. Se trata entonces con el conjunto de los modos de producción y de lectura de textos con los que una sociedad (o un sector dentro de ella) delimita, en el momento histórico de su vigencia, las fronteras discursivas que la diferencian con otras sociedades (o, en caso de tratarse de sectores, con otros dentro de la misma)" (Fernández, 1995: 4).

Hemos analizado discursivamente las publicidades de bebidas alcohólicas que fueron exhibidas en el período marzo-setiembre de 2014 para conocer y comprender el juego de recursos discursivos a partir del cual cada marca está buscando vincularse con su público real y potencial. Con el primero, para reafirmar su relación y fidelizarlos; con el segundo, apostando a generar algún tipo de vínculo, al menos en su memoria, que a futuro pueda traducirse en una práctica de consumo.

Para realizar el análisis de las publicidades se abordaron sus dimensiones retóricas, temáticas y enunciativas.

Para operativizar la observación de cada una de las dimensiones de análisis construimos un conjunto de elementos a trabajar. Ninguno de los elementos es tenido en cuenta en sí mismo como tampoco lo es cada dimensión aislada de las otras sino que es el sistema, la articulación de los elementos y procedimientos discursivos los que conforman el sentido en cada publicidad. Para aproximarnos a la dimensión retórica tuvimos en cuenta el uso de expresiones lingüísticas y entonaciones, los tipos de planos y encuadres, la duración de los planos, la velocidad del montaje, el uso de la música, la forma en la que se articula el ritmo de los planos y el de la música entre sí, las figuras retóricas utilizadas y los recursos gráficos. Para observar la dimensión temática nos interrogamos con qué sentidos se asocia el consumo de bebidas, cuáles son las formas creativas de la publicidad que se utilizan, qué tipo de situaciones se narran, cuáles son los personajes principales y los personajes secundarios, cómo son las locaciones, cómo están ambientadas, qué elementos se destacan, qué significa para esa publicidad ser "divertido", "exitoso", etc, cuáles son los lugares comunes y los razonamientos evocados y cuál es la promesa del producto. Lo enunciativo refiere a la construcción en el discurso de una situación comunicacional entre las figuras textuales de quienes emiten el mensaje, la marca, el producto, un personaje: enunciador/es, de quienes los reciben, los enuniciatarios (Steimberg, 1993), así como la relación que se construye entre ambos en el discurso: asimétrica> informativa, asimétrica>de autoridad, cómplice, etc.

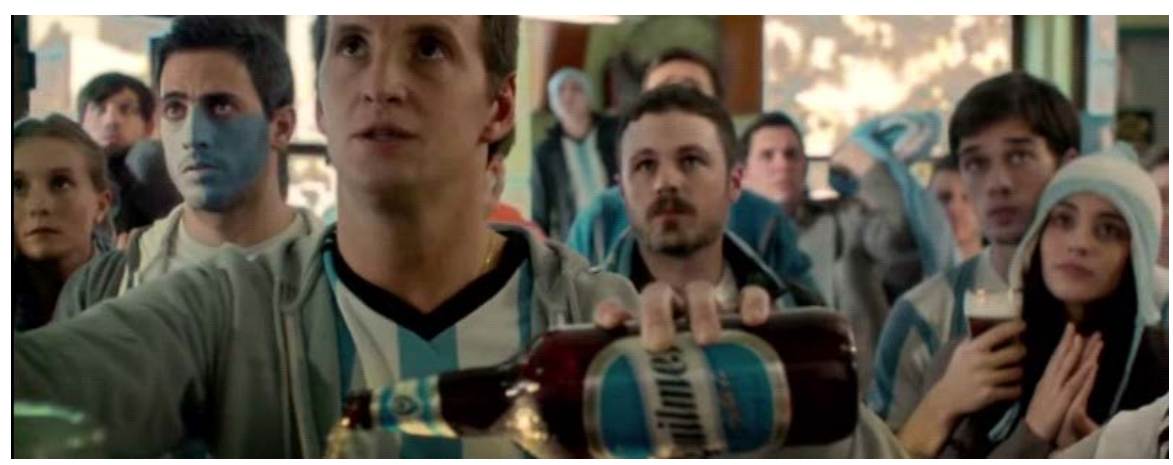




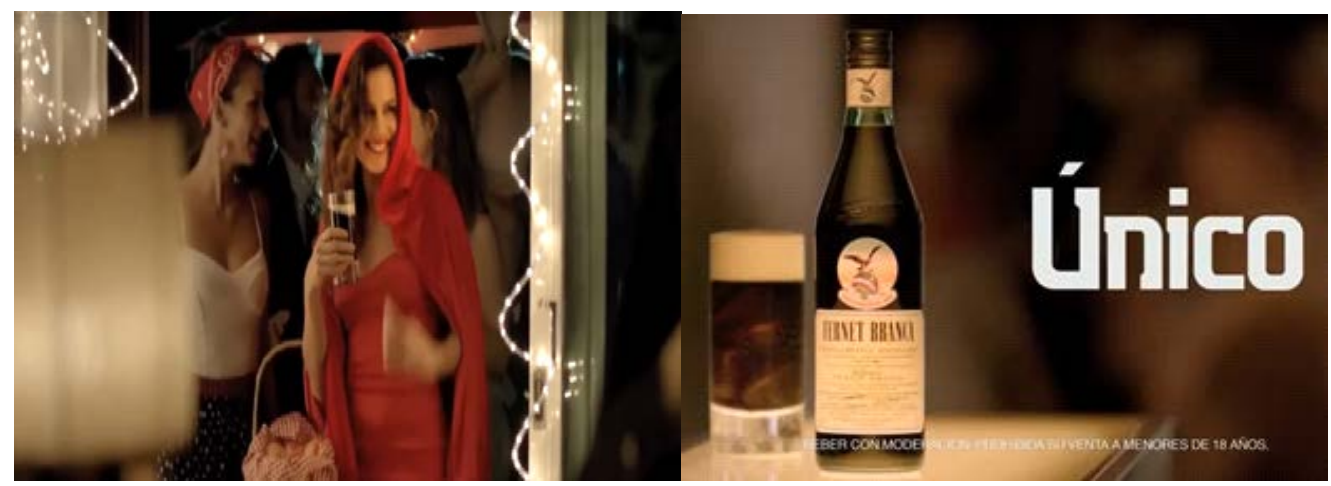

Del análisis de cada marca se desprende una serie de invariantes. En base a ellas se realizó el diseño de la guía de los grupos focales elaborada en relación al análisis de conceptos y categorías propuestas por las publicidades de bebidas alcohólicas.

La primera conclusión a la que se ha arribado es la omnipresencia de la propuesta del consumo de alcohol en los espacios de tiempo libre y de nocturnidad. Las motivaciones que proponen el consumo del producto, sea ya sea licor, cerveza o aperitivos, no se centran en las calidades o cualidades del mismo, sino en formas de identificación de la marca con un tipo de consumidor.

Ello implica un potente refuerzo a la naturalización del consumo de alcohol en toda instancia de ocio o tiempo libre en la que se realicen actividades compartidas con grupos de amigos, tanto como la música y la promesa de diversión.

Este rasgo tiene implicancias en las formas que se interpela a los destinatarios dado que los discursos plantean que el consumo de alcohol es necesario para la diversión y la integración en distintos grupos sociales con el riesgo de generar una estigmatización del no consumidor. Se constituye una sociedad de consumo que integra a todas y todos los consumidores y excluye a quienes no desean consumir.

Es interesante destacar que las publicidades apuntan a reforzar la identificación y afinidad con la marca. De esta manera, los procedimientos discursivos empleados en cada caso, van configurando un horizonte de expectativas que responde y resignifica los valores simbólicos compartidos, tales como: la amistad, el placer de compartir, la fiesta, comunidades de afinidad. Asimismo cabe mencionar los lugares comunes representados y en consecuencia, a lo que se asocia el ser joven: el sabor del encuentro, el disfrute con amigos, la actitud de vivir el momento, jugársela por los amigos, etc. Es en este marco en el cual no se representan situaciones de conflictividad social, ni de responsabilidad, como la escolaridad o lo laboral.

\section{Análisis de fanpages de Facebook}

La cultura de la convergencia le otorga a los receptores un rol de productores de mensajes mediatizados como no había ocurrido en las etapas anteriores de la historia de los medios de comunicación. El networking implica la multiplicación de nodos emisores. Como afirma Carlón "los usuarios desde que abren sus 'páginas personales' en Facebook se convierten en medios de comunicación" (Carlón, 2012: 182). 


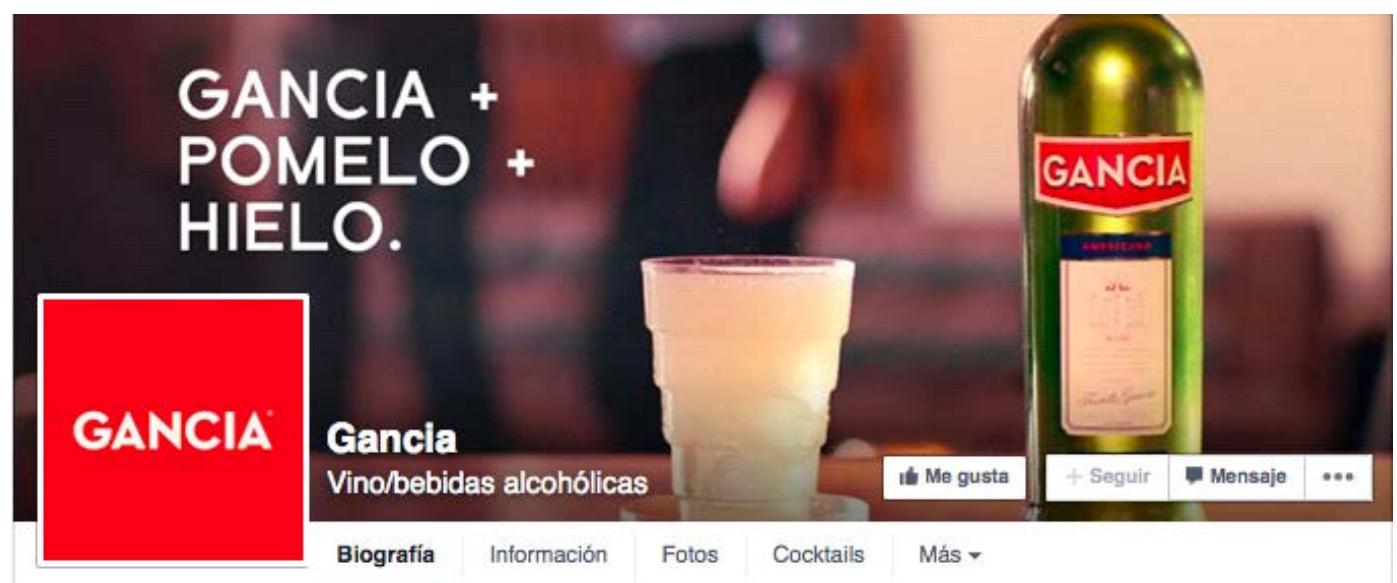

Q6 Qullmes Cerveza

Bariloche \#Invierno2014 \#Quilmes

Me gusta - Comentar - Compart

A3 A 1150 personas les gusta esto.

(1) Compartida 96 veces

He Ficardo Santos: Isso é Argentina, isso é

Q. cerveja...

Ver traducción

E.

Martin Santaella Oocohhh nectar de los dioses

$29 \mathrm{~h} \cdot$ Me gusta

2. Peter Frampton aguante la brahma

$23 \mathrm{~h} \cdot$ Me gusta

D. Walter Stella Me mata mal está CERVEZA.

$23 \mathrm{~h} \cdot$ Me gusta

젓 Norma Peralta Esta rerere buena aguante 1

quilmes

$23 \mathrm{~h} \cdot$ Me gusta

F. Andy Larky Seeeeeee

R2 $23 \mathrm{~h} \cdot$ Me gusta

1. Luis Pino Mmmmn cerveza

2. $23 \mathrm{~h} \cdot$ Me gusta

2 Claudia Alejandra Cacillas La más rica

$23 \mathrm{~h} \cdot$ Me austa

Escribe un comentario.
Matias Grilli ustedes con esas fiestitas con cerveza lo unico que van a lograr que me separe јajaja

$21 \mathrm{~h}+$ Me gusta

7. Marcos Moriena chomasuu

$20 \mathrm{~h} \cdot$ Me gusta

07. Claudia Haydee Migone Hay pq no me quede en esa edad y seguir disfrutando esos lindos boliche y esa rica cervezita $20 \mathrm{~h}$ - Me gusta

Jose Luis Escalera Una.maza $19 \mathrm{~h}$ - Me gusta

76. Ramon Oorlando Gramajo Hamas d uno d mis

2.2. amigos les gustaria tomarse una en este momento jajaja

$19 \mathrm{~h}$ - Me gusta

1d Mario Gauna Qué te gusta la cerveza o propaganda seguro la cerveza jajaja $18 \mathrm{~h} \cdot$ Me gusta

7. Beto Ramirez Siempre quise un comedor asi en casa. $17 \mathrm{~h}$ - Me gusta

F.7. Luis Ruben Peralta Es un asco la quilmes el que sabe sabe hace años q no es la misma $17 \mathrm{~h} \cdot$ Me gusta - 01

Pablo Cobos Quilmes cerveza de negros!! $17 \mathrm{~h}$ - Me gusta

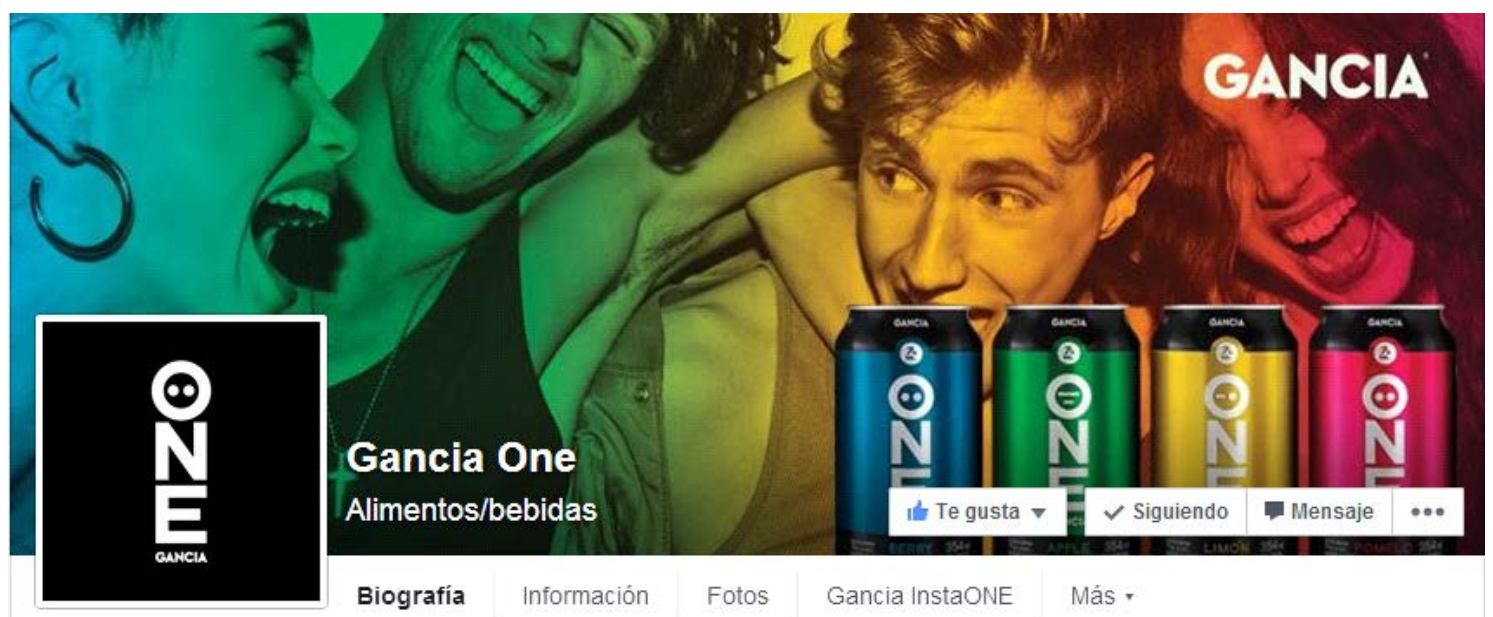




\section{Invariantes análisis fanpages}

Como se dijo, la inclusión de las fanpages en nuestro estudio surge del escenario transmediático de la comunicación de marca actualmente. Desde ese punto de vista, encontramos en las publicaciones de las cinco marcas analizadas referencias a temas ya detectados en las publicidades televisivas.

Se construyen el mundo de la socialidad, la noche, el fin de semana, la diversión y el encuentro entre amigos, aunque cada una se apoya más claramente en alguno de esos campos semánticos y lo hace con diversos tonos. Todos ellos, sin embargo, conforman uno que podemos denominar: la bebida en situación, entramada en la práctica misma del consumo. Así se suceden en videos y fotografías las escenas de grupos de amigos en boliches o bares compartiendo un trago o una cerveza. Allí la bebida alcohólica está presente para alentar la conquista y la seducción de un/a compañero para disfrutar la ocasión.

\section{Grupos focales}

A partir del análisis de las publicidades y páginas de Facebook se construyó una serie de interrogantes a responder en los grupos focales implementados en cada provincia:

- La diferencia nocturna/diurna: ¿La dicotomía día-noche está vinculada al "disfrute" nocturno versus lo "diurno" y la actividad productiva? ¿Las formas de habitar la noche y el día son diferentes?

- La diferencia semana/fin de semana: ¿Se asocia la semana a la actividad controlada ligada al "deber ser" (estudiar, trabajar) en contraposición a un fin de semana ligado al descomprimir y la actividad descontrolada?

- El desenfreno del "ser joven" y el consumo de alcohol: ¿Por ser "joven" se consume alcohol y se "proyectan" actividades de "levante"? ¿Siempre se es heterosexual cuando se está de levante en un boliche? ¿Cómo se alude a hombres y mujeres adultos en las publicidades?

- La asimetría ante el consumo: Cuando se sale con amigos y amigas, ¿todos toman lo mismo como promueven las publicidades o se toman bebidas diferentes? ¿Por qué esto sucedería o no? ¿qué dicen los amigos y amigas si no se consume alcohol?

- Ser urbano y consumir en la ciudad: Si en las publicidades sólo aparecen jóvenes del ámbito urbano. ¿Cómo es el consumo en ambientes rurales o semi rurales? ¿Cómo se comparten los momentos entre pares? ¿Qué formas toman esos momentos?

- Géneros y consumo de alcohol: ¿Cómo sé piensan los jóvenes como adultos? ¿Qué sentido le dan a la ingesta de alcohol? ¿cuáles son los roles y estereotipos de género implicados en la situación de consumo de alcohol? al varón que no consume alcohol o consume poco, ¿alguien lo enjuicia? ¿y a la mujer?

- La distinción y el consumo: ¿La ingesta de unas bebidas y no de otras otorga una mirada valorativa distinta? ¿Eso es percibido como distinción?

- El consumo de bebidas alcohólicas y el rito de pasaje a la adultez: ¿Bajo qué formas los jóvenes se auto perciben como "adultos" a través del consumo?

Una vez identificadas las dimensiones salientes del análisis se generaron las guías de pautas según los subgrupos definidos, 13 a 17 y 18 a 25 años. En las 
mismas se estableció la manera de intervenir en la discusión por parte del moderador al coordinar los focales.

En relación a los grupos focales, cabe destacar que se diseñaron dos dinámicas para generar un espacio propicio para la indagación de las prácticas y representaciones juveniles en torno a lugares, momentos, instancias y prácticas asociadas al consumo de alcohol como la diversión, el fin de semana y el encuentro con amigos.

En la primera de ellas, el "mapa de la diversión", se propuso trabajar en grupos para la descripción de lugares, situaciones y actores relacionados con el consumo de alcohol un fin de semana desde dos perspectivas: una real y otra ideal. De esta manera se apuntó a indagar tanto desde el relato de las prácticas concretas y cotidianas de los jóvenes como de sus expectativas e imaginarios en torno a ellas.

La segunda actividad, "visionado de imágenes", tuvo por finalidad relevar las representaciones de los jóvenes en relación a la publicidad de las bebidas alcohólicas, las formas y situaciones de consumo de alcohol y el valor simbólico de las publicidades y las marcas.
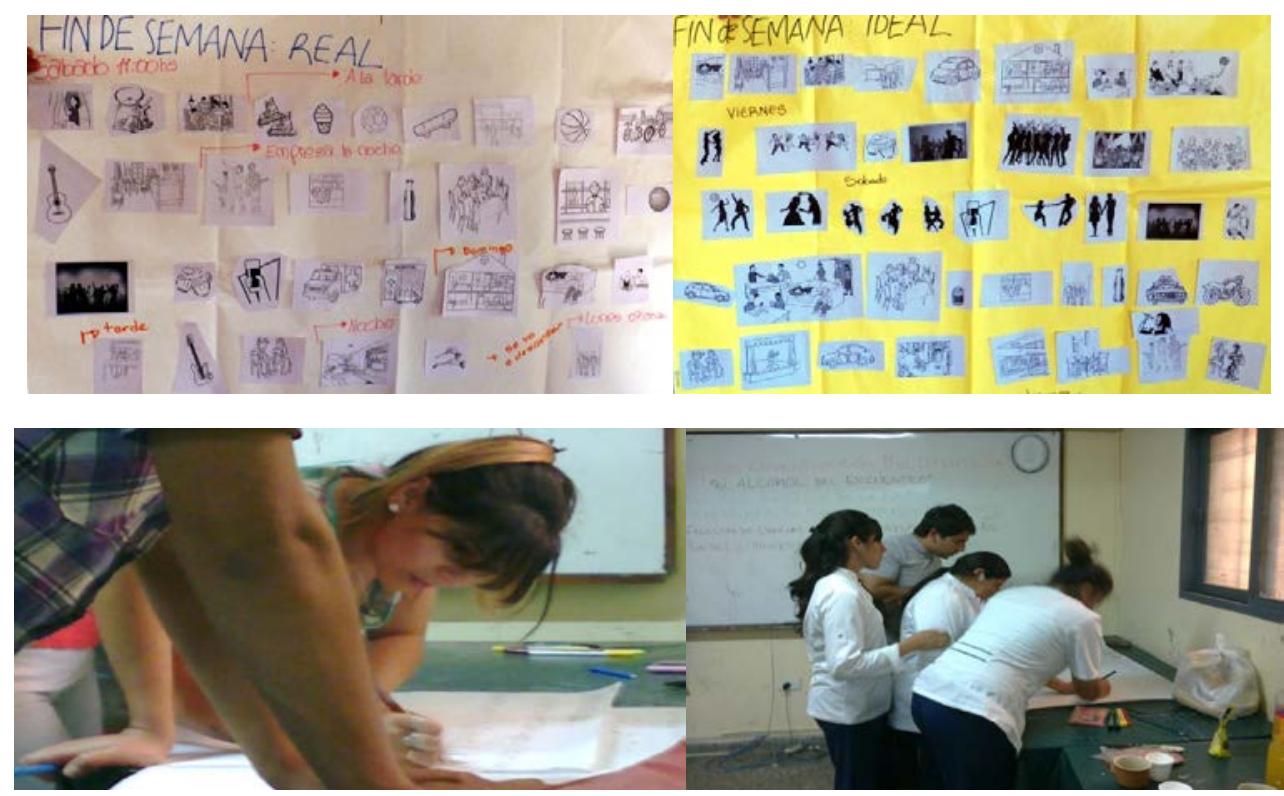

\section{Conclusiones focales}

El análisis de lo expresado por los jóvenes en los grupos focales permite afirmar que hay aspectos recurrentes y series semánticas que se van cruzando respecto del consumo de bebidas con alcohol en las percepciones de los jóvenes en todas las provincias donde se implementó el estudio. Por ejemplo, la alta valoración de la socialidad entre pares, donde los amigos, que ven diariamente, son figuras permanentes en la vida de jóvenes; la organización de la semana en torno a obligaciones asociadas a labores productivas como el estudio y el trabajo; aunque es importante señalar que en la muestra no hubo sujetos sin escolarizar; y la dedicación del fin de semana a la relajación, el encuentro con amigos, el disfrute y la diversión en contraposición al tiempo productivo de las responsabilidades.

La mayor diferencia en el uso de ese tiempo libre se encuentra en la forma en que se piensa e invierte el mismo. Los adolescentes lo hacen desde la libertad y la diversión mientras que los jóvenes adultos desde el descanso. En ese disfrute y 
diversión asociados al fin de semana y el tiempo libre se evidencian dos esquemas de funcionamiento intensamente marcados y repetidos de manera similar en cada una de las provincias relevadas. Por un lado, un esquema diurno que incluye paseos, encuentros familiares y la realización de deportes al aire libre. Por otro lado, un esquema de salidas nocturnas que comienza con una previa, en la mayoría de los casos incluye juntarse a comer y se extiende hasta entrada la noche con salidas a boliches o a recorrer distintos espacios en horarios que no son habitados por los adultos. La nocturnidad aparece, de esta forma, como un espacio de sustracción del mundo adulto.

\section{CONCLUSIONES FINALES}

Considerando el análisis del entrecruzamiento entre el discurso de las marcas y el de los jóvenes, podemos afirmar una circularidad entre las propuestas de las marcas y las representaciones de los jóvenes. Tal como hemos observado a la largo de la investigación, a través de las distintas "realidades" relevadas en los focales, las opciones de consumo propuestas por las marcas son recibidas y reinterpretadas, pero en todas ellas es patente el poder de naturalización de la propuesta de consumo. En ella se propone una diferenciación y distinción a través de la práctica cultural de consumir una marca en particular, siempre regulada por las ofertas del mercado. Tal como hemos visto en el apartado del análisis de publicidades el alcohol siempre está presente en la práctica juvenil de diversión y nocturnidad, no se venden atributos y beneficios del alcohol, en tanto producto, sino que ya están establecidos.

Es importante destacar que en el caso de los adolescentes de 13 a 17 años, la iniciación en el proceso de ingesta de alcohol está vinculada a la búsqueda de sensaciones nuevas que coinciden con sus discursos relacionados con el desafío, la transgresión. El momento de las primeras salidas nocturnas coincide con el inicio del consumo regular de alcohol.

Este rasgo tiene implicancias en las formas que se interpela a los destinatarios dado que los discursos plantean que el consumo de alcohol es necesario para la diversión y la integración en distintos grupos sociales con el riesgo de generar una estigmatización del no consumidor. Se constituye una sociedad de consumo que integra a todas y todos los consumidores y excluye a quienes no desean consumir.

Responder nuestra pregunta sobre las disposiciones al consumo que generan las publicidades de marcas de bebidas alcohólicas nos obliga a pensar un punto de partida de la generación de esas disposiciones. Allí es donde habría que intervenir desde las políticas públicas para crear las condiciones para un cambio de hábito en el consumo de alcohol de los jóvenes.

Si las marcas están representando ciertas prácticas propias de los jóvenes hoy: las marcas nos llevan de vuelta a los jóvenes. ¿Entonces tenemos la causa y el efecto en el mismo punto? ¿Cómo ese funcionamiento de las marcas como "espejo" está generando disposiciones al consumo?

Como se planteó en las conclusiones preliminares del análisis de las piezas publicitarias, la relación que se propone entre marca y consumidor es de identificación, a partir de la representación de las prácticas de los jóvenes en los discursos de las marcas vía procedimiento slice of life. Esta forma creativa implica la representación de una etapa de la vida, en este caso, la juventud, con las prácticas y valores que conlleva a nivel identitario dicha categoría socio-cultural. 
Así las fiestas, la diversión, las situaciones de seducción, los momentos compartidos con amigos son los escenarios en los que la bebida alcohólica se ha entramado socialmente.

Este espacio significante es naturalizado por los jóvenes consultados. A partir de allí el consumo excesivo puede darse por juego, por búsqueda de límites o por desconocimiento, según plantean los jóvenes.

El mundo del consumo de alcohol construido por las marcas sintoniza en mayor medida con las prácticas del segmento de jóvenes menor (13-17) que se está iniciando en el consumo. La fiesta, la "joda" son mucho más habituales entre jóvenes que aún no trabajan.

El acceso restringido al dinero - sobre todo de los adolescentes - hace que las situaciones de consumo de los jóvenes sean mucho menos glamorosas que las planteadas por las marcas, sin embargo encontramos dos conceptualizaciones sobre el consumo de bebidas alcohólicas presentes tanto en los discursos de las marcas como en los de los jóvenes con respecto a su práctica. Se trata de dos espacios de conexión entre ambos conjuntos discursivos que pueden estar funcionando como generadores de disposiciones para el consumo.

El primer núcleo conceptual que llamamos 'placer de la ingesta', puede alojarse en la bebida misma o en las consecuencias de su consumo. Es decir, beber por una motivación más hedonista o más pragmática. Los jóvenes consultados manifestaron ambas motivaciones, pero fueron los mayores (18 a 25 años) quienes vincularon su consumo de alcohol con el gusto por el sabor de la bebida. Este foco en la bebida misma se presentó como propio de los consumidores con algunos años de experiencia y ciertas preferencias ya establecidas. En cambio, los jóvenes de 13 a 17 años asociaron su consumo mayormente a estados y situaciones posteriores.

Las marcas, por su parte, recurren a ambos polos del placer de la ingesta: la bebida o sus consecuencias. Con cierta preeminencia del primer polo en la subcategoría vinos y apertitivos, y de la segunda en la subcategoría cervezas y los restos del vino transformados en bebida carbonatada tales como frizze. Sin embargo, esto no es lineal, hay casos cruzados como la cerveza Schneider que tematiza el origen de su materia prima.

El segundo núcleo conceptual está vinculado al anterior pero enfoca al consumidor, no al producto. En el 'juego que propone la ingesta' de la bebida, ¿cuál es el rol que asume el consumidor? Otra vez la respuesta puede encontrarse entre dos polos: un consumidor autocontrolado que elige su bebida e incluso la prepara y un consumidor sujeto a sus circunstancias, que bebe lo que le toca, lo que elige el grupo, lo que hay que tomar. La figura del consumidor autocontrolado aparece en prácticas manifestadas por jóvenes del segmento 18 a 25 años y también entre los aperitivos como Gancia o Cinzano. Ambos discursos - de estas marcas y de estos jóveneshablan de preparar la bebida, elegir los ingredientes y hasta de la forma en que debe lucir el vaso.

En el otro extremo el consumidor sujeto de sus circunstancias, en algún sentido un consumidor gregario, se presenta en las narraciones de los jóvenes adolescentes que están incorporándose en el consumo de bebidas alcohólicas. Del lado de las marcas son las cervezas las que recurren con mayor frecuencia a esta figura.

En síntesis, el análisis comparativo de los discursos de las marcas y de los jóvenes nos permitió identificar una tendencia a la siguiente relación en el campo semántico del consumo de bebidas alcohólicas: 


\begin{tabular}{|c|c|c|c|}
\hline Consumidor & Bebida & Jóvenes & Subcategorías \\
\hline Autocontrolado & Producto (sabor) & $+18-25$ & + vinos /aperitivos \\
\hline Gregario & Efectos & $+13-17$ & + cervezas \\
\hline
\end{tabular}

Por ello consideramos que este trabajo es un interesante aporte a la discusión en torno a los avances en cuanto a la protección de los derechos de niños, niñas y adolescentes. Lamentamos la anulación de la la Ley de Servicios de Comunicación Audiovisual № 26.522 que daba marco a las acciones del Estado en el marco de las defensorías y observatorios de medios y publicidad para garantizar el acceso a información completa para la toma de decisiones, así como para aportar al desarrollo de políticas y acciones comunicacionales preventivas y de promoción de la salud ligadas a la perspectiva de atención primaria de la salud.

\section{Bibliografía}

- Ancery, P. (2005) "La cerveza, el hit del momento", disponible en http://www.adlatina.com/adlatina-magazine/adlatina-magazine-15.

- Boletín de Vigilancia Enfermedades No Transmisibles y Factores de Riesgo Descripción epidemiológica de la morbimortalidad (2014) por lesiones de tránsito en Argentina.

- Capriotti, P. (2009). Branding Corporativo. Chile: Libros de Empresas.

- Carlón, M. (2012) "En el ojo de la convergencia. Los discursos de los usuarios de Facebook durante la transmisión televisiva de la votación de la ley de matrimonio igualitario", en Carlón, M. y Fausto Neto A. (comps.) Las políticas de los internautas, Buenos Aires, La Crujía.

- Chaves, Mariana (2007) "Salir de noche: ejercicios de autonomía juvenil en tiempos y espacios nocturnos" en: Revista Acceso Directo No 2. Rosario: Dirección de Juventud, Municipalidad de Rosario. pp.99-119

- Consejo Municipal de la Salud (2015): Informe Sociodemográfico, Dirección de Salud Mental de Villa María, Villa María.

- Córdoba, María Liliana y Morales, Susana (2013): Aportes para el desarrollo de la televisión digital abierta. Informes de $1+D$ del Programa Polos Audiovisuales Tecnológicos, Ministerio de Planificación Federal, Inversión Pública y Servicios.

- Dodaro (2012) Sobre el saber médico y el (o los) discurso(s) hegemónico(s) ¿Cambio de perspectiva o cambio de significantes?, Mesa de Comunicación y Salud, Jornadas Carrera Ciencias de la Comunicación, FSOC, UBA.

- Fernández, J.L. (1995) "Estilo discursivo y planeamiento comunicacional", en Oficios Terrestres №1, La Plata, Facultad de Periodismo y Comunicación Social, UNLP.

- Fernández, J.L. (2014) "Periodizaciones de idas y vueltas entre mediatizaciones y músicas", en Fernández, J.L. (coord.) Postbroadcasting. Innovación en la industria musical, Buenos Aires, La Crujía.

- Fuentes, G (2011). "Plan de Comunicación 2.0" disponible en http://www.reddircom.org/pdfs/Revista\%20Imagen\%20y\%20Comunicacion\%20N18.pdf 
- Jenkins, H., (2008) "Adoración en el altar de la convergencia: Un nuevo paradigma para comprender el cambio mediático", en Convergence Culture. La cultura de la convergencia de los medios de comunicación. Barcelona, Paidós Ibérica.

- Kornblit A., MendesDiz A., Adaszko D. Salud y Enfemedad desde la Perspectiva de los Jóvenes. Documento No 47 del Instituto Gino Germani

- Kornblit, A. L. y Adaszko, D. (2008) "Violencia y discriminación en el ámbito de la escuela media". En VVAA, Investigaciones por la diversidad. Publicación de los trabajos distinguidos con el Premio a la Producción Científica sobre Discriminación en la Argentina. Buenos Aires: INADI.

- Kornblit, A. L., MendesDiz, A. M. y Adaszko, D. (2006) Salud y enfermedad desde la perspectiva de los jóvenes. Un estudio en jóvenes escolarizados de todo el país. Documento de Trabajo No 47, IIGG, Facultad de Ciencias Sociales, UBA. Biblos.

- Kornblit, Ana Lía (2007) "Metodologías cualitativas en ciencias sociales", Ed.

- Laespada, M. T. Y J. Pallarés Gómez (2001) Informe sobre Juventudes. Revista de Estudios de Juventud. Instituto de la Juventud. Septiembre 2001. España. ISSN 02114364.

- Laespada, M. T. Y J. Pallarés Gómez (2001) Informe sobre Juventudes. Revista de Estudios de Juventud. Instituto de la Juventud. Septiembre 2001. España. ISSN 02114364. da Moeda.

- Machado Pais, José (2003) Culturas juvenis. Lisboa: Imprensa Nacional-Casa

- Marafioti, Roberto (2003) “Los patrones de la argumentación”. Ed. Biblos.

- Margulis, M. (comp.) (1994) La cultura de la noche. Vida nocturna de los jóvenes en Buenos Aires. Buenos Aires: Espasa Calpe.

- Mata, María Cristina (1997): Públicos y consumos culturales en Córdoba, Centro de Estudios Avanzados, Universidad Nacional de Córdoba, Córdoba.

- MendesDiz A. y Di Leo P. Me miró mal. Violencia y discriminación en las salidas nocturnas de jóvenes de tres ciudades argentinas. (2009) Síntesis Forense (en prensa)

- Ministerio de Salud de Nación, Dirección Nacional de Salud Mental y Adicciones, 2011. Lineamientos normativos para la atención integral de la población frente al consumo excesivo de alcohol y otras sustancias psicoactivas Normativas publicadas en http://www.msal.gov.ar/saludmental/index.php/informacion-paraequipos-de-salud/publicaciones

- Observatorio Integral de la Región (OIR) - Instituto de Extensión, Universidad Nacional de Villa María (UNMV): Encuesta Anual de Hogares Urbanos (EAHU-INDEC) - Aglomerado Villa María-Villa Nueva - Operativo 2013 (Tercer Trimestre), Villa María, 2014.

- Observatorio Integral de la Región (OIR) - Instituto de Extensión, Universidad Nacional de Villa María (UNMV): Censo Nacional de Población, Hogares y Viviendas 2010 - INDEC. Resultados definitivos de población para la provincia y el Dpto. General San Martín, Mayo 2013. 
- Plan Nacional de Salud Mental. Disponible en: http://www.msal.gov.ar/saludmental/index.php?option=com content HYPERLINK "http://www.msal.gov.ar/saludmental/index.php?option=com content\&view=article\&id= 247:se-aprobo-el-plan-nacional-de-salud-mental\&catid=4:destacados-slide247"\& HYPERLINK "http://www.msal.gov.ar/saludmental/index.php?option=com content\&view=article\&id= 247:se-aprobo-el-plan-nacional-de-salud-mental\&catid=4:destacadosslide247"view=article HYPERLINK "http://www.msal.gov.ar/saludmental/index.php?option=com content\&view=article\&id= 247:se-aprobo-el-plan-nacional-de-salud-mental\&catid=4:destacados-slide247"\& HYPERLINK

"http://www.msal.gov.ar/saludmental/index.php?option=com content\&view=article\&id= 247:se-aprobo-el-plan-nacional-de-salud-mental\&catid=4:destacadosslide247"id=247: se-aprobo-el-plan-nacional-de-salud-mental HYPERLINK "http://www.msal.gov.ar/saludmental/index.php?option=com content\&view=article\&id= 247:se-aprobo-el-plan-nacional-de-salud-mental\&catid=4:destacados-slide247"\& HYPERLINK "http://www.msal.gov.ar/saludmental/index.php?option=com content\&view=article\&id= 247:se-aprobo-el-plan-nacional-de-salud-mental\&catid=4:destacadosslide247"catid=4:destacados-slide247

- Reguillo, Rossana (2000) Emergencia de culturas juveniles. Estrategias del desencanto. Buenos Aires: Norma.

- Ribes, X. (2007) "La Web 2.0. El valor de los metadatos y de la inteligencia colectiva", en Revista Telos No 73, octubre - diciembre 2007. Disponible en: http://telos.fundaciontelefonica.com/telos/articuloperspectiva.asp@idarticulo=2 HYPERLINK "http://telos.fundaciontelefonica.com/telos/articuloperspectiva.asp@idarticulo=2\&rev=7 3.htm"\& HYPERLINK "http://telos.fundaciontelefonica.com/telos/articuloperspectiva.asp@idarticulo=2\&rev=7 3.htm"rev=73.htm

- Schwarz, P. K. N. y MendesDiz, A. M. (2009) "Abordaje teórico y sociohistórico de las transformaciones de la familia. Su realidad actual desde la mirada de los jóvenes". Ponencia presentada en Segundas Jornadas Nacionales de Historia Social. La Falda, Córdoba.

- SCOLARI, Carlos (2008): Hipermediaciones. Elementos para una Teoría de la Comunicación Digital Interactiva, Barcelona: Gedisa.

- Semprini, A. (1995) El marketing de la marca. México. Paidós

- Silba, Malvina (2007) "El baile de las pibas, las piñas de los pibes (o viceversa): sobre feminidades y masculinidades en jóvenes de sectores populares" Ponencia presentada en $1^{\text {a }}$ Reunión Nacional de Investigadoras/es en Juventudes. La Plata, UNLP. Gedisa.

- Simmel, G. (2002) Cuestiones Fundamentales de sociología. Barcelona:

- Sistema de Información Cultural de la Argentina (2014): Encuesta de consumos culturales y entorno digital. Informe 2: Computadora, internet y videojuegos e Informe 3: Audiovisual, Dirección Nacional de Industrias Culturales, Ministerio de Cultura de la Nación. 
Disponibles en: http://sinca.cultura.gob.ar/sic/encuestas/archivos/videojuegos-01a4.pdf y http://sinca.cultura.gob.ar/sic/encuestas/archivos/audiovisual-01-a4.pdf

- Sznaider, B., Tobi, X. (2012) "Instituciones en la red: cuando el público se hace usuario" (inédito). Disponible en: https://es.scribd.com/doc/116701859/Instituciones-enla-red-cuando-el-publico-se-hace-usuario-Entre-los-discursivo-y-la-accion-work-inprogress

- Steimberg, O. (1993) "Semiótica de los medios masivos". Ed. Atuel, 1993

- Urresti, M. (2007) "Transformaciones de la nocturnidad" en: Revista Acceso Directo No 2. Rosario: Dirección

- Verón, E. (1985) "El análisis del "contrato de lectura": un nuevo método para los estudios de posicionamiento en los soportes de los media", En Les médias: expériences, recherchesactuelles: aplications. IREP, Paris. (Traducción Lucrecia Escudero).

- Verón, E (1987) La Semiosis Social, Barcelona, Gedisa

- VERÓN, E. (1995): "Semiosis de lo ideológico y del poder", en Semiosis de lo ideológico y del poder. La mediatización. Buenos Aires: Facultad de Filosofía y Letras, Universidad de Buenos Aires (UBA).

- Vigarello, G. (2005) Historia de la belleza. El cuerpo y el arte de embellecer desde el Renacimiento hasta nuestros días, Nueva Visión, Buenos Aires.

- Toffler, A. (1980). La Tercera Ola, Bogotá, Colombia, Plaza \& Janes Editores.

Datos de Mortalidad actualizados al 2012. Datos de Morbilidad hospitalaria actualizados al 2011.

A.N.M.A.T. (Administración Nacional de Medicamentos, Alimentos y Tecnología Medica) Código Alimentario de bebidas espirituosas, alcoholes, bebidas destiladas alcohólicas y licores, disponible en: http://www.anmat.gov.ar/alimentos/codigoa/CAPITULO XIV.pdf y Código alimentario de bebidas fermentadas disponible en: http://www.anmat.gov.ar/alimentos/codigoa/Capitulo XIII.pdf

Información estadística

Estudio Nacional en población de 12 a 65 años, sobre consumo de sustancias psicoactivas, Argentina 2010

Plan Estratégico de Seguridad Vial para Motovehículos, Agencia Nacional de Seguridad Vial, Dirección Nacional de Observatorio Vial, durante el año 2011

Quinto estudio de uso indebido de drogas y la consulta de emergencia (publicado en Argentina Febrero 2013)

Encuesta Nacional EnPreCoSP e INDEC

Encuestas de Factores de Riesgo

Encuestas de Salud Escolar

MSAL

SEDRONAR Nación.

Encuesta Nacional de Consumos Culturales, SinCA, Secretaría de Cultura de la 
Irigaray, F. et al (2014): Estudio Rosarinos frente a las pantallas, Dirección de Comunicación Multimedial de la Universidad Nacional de Rosario. Disponible en: http://www.unrinteractiva.com.ar/2014/07/rosarinos-frente-a-las-pantallas/

\section{Artículos consultados}

\section{Artículos de diarios y sitios web de noticias}

21-09-2014, La Nación: "Más y más litros_las bebidas alcohólicas _listas para tomar_ ya son furor _ en el - país", http://Www.lanacion.com.ar/1728936masymaslitroslasbebidasalcoholicaslistasparatom aryasonfurorenelpais, consultado 31-01-2015.

26-09-2014, BAE Negocios: "Aperitivos a toda hora", http://www.diariobae.com/notas/34355-aperitivos-toda-hora.html, consultado 31-012015.

13-02-2012, ADLatina: "BBDO presenta su primer trabajo para GanciaOne", http://www.adlatina.com/publicidad/bbdopresentasuprimertrabajoparaganciaone, consultado 31-01-2015.

13-11-2009, ADLatina: "One, preestreno de CraveroLanis para Gancia", http://www.adlatina.com/publicidad/onepreestrenodecraverolanisparagancia, consultado 31-01-2015.

04-12-2013. ADLatina; "GanciaOne renueva su identidad visual", http://www.adlatina.com/breves/gancia-one-renueva-su-identidad-visual, consultado 31-01-2015.

06.09.2013, ADLatina: "Reposicionar una gran marca es el trabajo más difícil", http://www.adlatina.com/negocios/\%E2\%80\%9Creposicionar-una-gran-marca-es-eltrabajo-m\%C3\%A1s-dif\%C3\%ADcil\%E2\%80\%9D, consultado 31-01-2015.

http://onaf.uda.edu.ar/tendencias-de-consumo-cultural-en-adolescentes/

Visitada el 18 de abril de 2015.Sananes A. y Trameur Y. "Jóvenes, Internet y Ciudadanía: una propuesta de educación digital, medio ambiental, en el marco de la educomunicación para el cambio social". Tesina de grado. Carrera de Comunicación Social. Facultad de Ciencias Políticas y Sociales. Universidad Nacional de Cuyo. Noviembre de 2013.

22-06-2014. El diario del centro del país: "Desocupación en mujeres duplica la de los varones" http://www.eldiariocba.com.ar/noticias/nota.asp?nid=82926 Consultado el 27/03/2015

Quinta Encuesta Nacional a Estudiantes de Enseñanza Media 2011 Informe Final de Resultados, disponible en http://portal.educacion.gov.ar/files/2013/10/adiccion quinta encuesta.pdf, visitada el 5 de abril de 2015.

http://www.ciudaddemendoza.gov.ar/,visitada el 3 de abril de 2015.

Ancery, P. (2005) "La cerveza, el hit del momento", disponible en http://www.adlatina.com/adlatina-magazine/adlatina-magazine-15, consultada el 14 de abril de 2015.

Sitio web oficial de la Municipalidad de Villa María: "La ciudad". http://www.villamaria.gov.ar/secciones/ciudad - Consultado el 24/03/2015

\section{Cómo citar el artículo}

Dodaro, C. (2016). "Decime quien consume a quien". Estudio sobre la creación de disposiciones al consumo de bebidas con alcohol generadas por la publicidad en jóvenes. Revista de Comunicación y Salud, Vol. 6, pp. 55-69.

DOI: http://doi.org/10.35669/revistadecomunicacionysalud.2016.6.55-69 